\title{
CÓMO COMPRENDER LOS RECLAMOS DE LOS PUEBLOS INDIOS
}

\author{
Agustín Pérez Carrillo \\ Universidad Autónoma Metropolitana (México)
}

\section{Propósito}

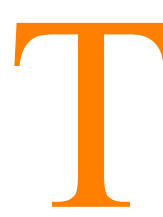

engo interés en darle contenido a la siguiente metáfora: más allá de la búsqueda del reconocimiento de la cultura y los derechos de los pueblos indios por medio de reformas constitucionales. Motivan mi interés la negociación entre el Ejército Zapatista de Liberación Nacional (EZLN) y el gobierno federal, así como los avances y retrocesos observados y las propuestas y contrapropuestas de reforma constitucional publicadas.

Tengo la percepción de que ningún contenido constitucional, aún el más favorable a la cultura y derechos de los pueblos indios, será suficiente para lograr la paz si subsisten las condiciones adversas para la eficacia de las reformas por no enfrentar las causas que originaron el conflicto social.

\section{Obstáculos. Identificaré algunos obstáculos que impiden la solución del conflicto social}

2.1 La incapacidad del gobierno federal para comprender el lenguaje de quienes defienden formas de vida tradicionales y exigen el reconocimiento de su cultura y sus derechos como pueblos indios. Este obstáculo tiene como consecuencia que las autoridades estatales han entendido, en sus marcos de referencia ideológicos, las reclamaciones de los pueblos indios, actitud con la cual se niega la posibilidad de comprender otras formas de vida y las justificaciones correspondientes. Se advierte en esa actitud una manifestación de intolerancia a sistemas de vida diferentes al aceptado por el gobierno, razón por la cual tampoco tiene disposición de otorgar facilidades para la subsistencia y desarrollo de las formas de vida reclamantes.

Dos frases de Wittgenstein en Los comentarios sobre La rama dorada, de Frazer destacan una actitud de incomprensión de formas de vida diferentes a las propias: «Frazer es incapaz de imaginar un sacerdote que no sea, en el fondo, un párroco inglés de nuestro tiempo con toda su estupidez y flaqueza.» «Frazer es mucho más salvaje que la mayoría de sus salvajes, ya 
que éstos no están tan lejos de comprender un asunto espiritual de como lo está un inglés del siglo veinte. Sus explicaciones de las prácticas primitivas son más burdas que el sentido de las prácticas mismas». ${ }^{1}$

2.2 Otro obstáculo es que no se ha abordado el debate filosófico entre la «universalidad» de la ética y la diversidad cultural. Quien sostendría la universalidad, en este caso el gobierno, es quien recibe la reclamación de reconocimiento de la pluralidad, de la eticidad de formas de vida; en consecuencia, es quien está obligado a avanzar en la búsqueda del consenso racional. ${ }^{2}$

2.3 Otro obstáculo es que uno de los negociadores, el gobierno, tiene la calidad de juez y parte; en consecuencia, toma decisiones y las instrumenta de acuerdo con su racionalidad y su poder.

2.4 El conflicto social existente a nivel de la nación mexicana, no asumirlo y no enfrentarlo con las medidas apropiadas son otros obstáculos para que la negociación carezca de resultados favorables al reclamo sobre cultura y derechos de los pueblos indios. En este contexto los reclamos de la Declaración de la Selva Lacandona, sus antecedentes y consecuencias son síntomas del conflicto social, no un problema aislado, en ámbitos locales y que lesiona a pequeños grupos a partir de un tiempo relativamente corto.

\section{Modelos para enfrentar los problemas sociales y concepto de autoridad}

Planteo la siguiente pregunta: ¿con qué autoridad ha actuado el gobierno federal en los hechos, en los procesos y en las tomas de decisión que se relacionan con las reclamaciones del EZLN? La respuesta permitirá evaluar sus actividades, criticarlas, predecir posibles comportamientos y conocer las expectativas para resolver el conflicto social o prolongarlo con los costos que probablemente se incrementarán. ${ }^{3}$ Para ese efecto, presentaré algunos modelos y ejemplos en decisiones o acciones del gobierno federal.

3.1 Modelos reactivos de poder. En estos modelos se ignora la complejidad de los problemas y se considera que las situaciones irregulares tienen solución en la ideología político-jurídica dominante.

${ }^{1}$ Wittgenstein, Ludwig, Comentarios sobre La rama dorada, ed. Rush Rhees, tr. Javier Esquivel, Universidad Nacional Autónoma de México, Instituto de Investigaciones Filosóficas. México, 1997, pp. 15 y 22.

${ }^{2}$ Cfr. Garzón Valdés, Ernesto, «Problema ético de las minorías étnicas», en Ética y diversidad cultural, León Olivé (Compilador), Universidad Nacional Autónoma de México y Fondo de Cultura Económica, S. A. de C. V., 1993. Olivé, León, «Multiculturalismo: ni universalismo, ni relativismo» en Filosofía moral, educación e historia, León Olivé y Luis Villoro, eds., Universidad Nacional Autónoma de México, 1996.

${ }^{3}$ Me orientaré fundamentalmente por John Burton en las exposiciones de su libro Conflict: Resolution and Prevention, The Macmillan Press Ltd, London, 1990. 
3.1.1 En el primer tipo la parte A percibe la situación problemática, la incorpora al ambiente de poder, como en una especie de caja negra, determina sus propósitos y conoce los de $\mathrm{B}$. La asignación de valores a los propósitos de $\mathrm{A}$ es $=+\mathrm{x}$ y los de $\mathrm{B}=-\mathrm{x}$, donde $\mathrm{x}$ $=1$. El modelo reacciona sólo en función de un resultado de ganancia-pérdida (win-lose) y la dinámica tiende a la obtención, por $\mathrm{A}$, de una decisión coactiva (settlement enforced).

El primer tipo de modelo reactivo de poder se actualiza en los siguientes casos:

Primer ejemplo.

La determinación por la Secretaría de Gobernación el 5 de enero de 1994, de cuatro condiciones que a continuación transcribo: «El cese de las hostilidades y agresiones a los pueblos y a las personas; la deposición y entrega de las armas, incluyendo los 1,500 kilogramos de dinamita robados el día último del año pasado a Pemex; la devolución de los rehenes y los secuestrados, y la identificación de los interlocutores y dirigentes del grupo

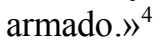

Segundo ejemplo.

Los nombramientos del Ejecutivo Federal de diversos comisionados para la paz en Chiapas. Ya son cuatro.

Tercer ejemplo.

La creación por el Ejecutivo de la Unión, el 19 de enero de 1994, de la Comisión Nacional de Desarrollo Integral y Justicia Social para los Pueblos Indígenas encargado de la satisfacción de algunas necesidades humanas. ${ }^{5}$

Cuarto ejemplo.

Las órdenes de aprehensión en contra de Rafael Sebastián Guillén Vicente (el gobierno lo identificó como Marcos), Jorge Javier Elorriaga Berdegué, Fernando Yáñez Muñoz, Jorge Santiago Santiago y Silvia Fernández Hernández por los delitos de terrorismo, conspiración y rebelión, anunciadas por el Dr. Ernesto Zedillo Ponce de León el 9 de febrero de $1995 .^{6}$

Quinto ejemplo.

La aprehensión de Fernando Yáñez Muñoz el 21 de octubre de 1995, identificado anteriormente por el Dr. Zedillo como el Comandante Germán del EZLN. Esta acción penal perturbó la negociación entre el EZLN y el gobierno federal y transgredió la Ley para el Diálogo, la Conciliación y la Paz

${ }^{4}$ Proceso No. 898/17 de enero/1994.

${ }^{5}$ Proceso No. 895 p. 17 y Proceso 902. pp. 34 y ss.

${ }^{6}$ Proceso No. 954, 13/febrero/1995 en el cual aparecen varios reportajes sobre el particular. Destaco que fue a propósito de declaraciones de Salvador Morales Garibay que el gobierno federal consideró que se había identificado a varios líderes del EZLN y que después ya no se supo de dicha persona y no hubo oportunidad de carearlos con los acusados. Causa 39/1/95 de la Procuraduría General de la República. 
Digna en Chiapas, según la cual se mantendrían suspendidos los procedimientos en contra de los integrantes del EZLN, la ejecución de las órdenes de aprehensión dictadas y las investigaciones por los hechos relacionados con la declaración de guerra, si se iniciaba y mantenía el diálogo para suscribir un acuerdo de concordia y pacificación. Como a Yáñez Muñoz se le había identificado oficialmente como Comandante Germán del EZLN, resulta obvia la violación legal. Se sabe que los delitos que se le imputaron fueron los de portación de armas prohibidas y contra la salud, sin embargo quedó libre el 27 del mismo mes y año, por sobreseimiento decretado a petición de la Procuraduría General de la República. ${ }^{7}$

Sexto ejemplo.

Una decisión para acabar con el conflicto armado es la guerra de baja intensidad sostenida por el Ejército Nacional Mexicano (ENM) en lugares estratégicos del Estado de Chiapas con el fin de desgastar al EZLN y desalentar a sus simpatizantes. En este tipo de medidas se advierte que no se pretende la solución del conflicto social y sí, por definición, prolongarlo y hacer improbable la satisfacción de las necesidades humanas fundamentales exigida por el EZLN. ${ }^{8}$

3.1.2 El segundo tipo del modelo reactivo de poder es un modelo cibernético de ensayo y error (Trial-and-error cybernetics) en cuya aplicación se determinan los propósitos de $\mathrm{A}$; se conocen los de $\mathrm{B}$ y se decide que el valor de los de $\mathrm{A}$ es $=+\mathrm{x}$ y los de $\mathrm{B}=-\mathrm{x}$, donde $\mathrm{x}>0.5$ Se trata también de un resultado de ganancia-pérdida aunque en proporción menos extrema y la decisión que toma A que considera los propósitos de $\mathrm{B}$, es una decisión coactiva.

Como actualizaciones del segundo tipo del modelo reactivo de poder menciono las siguientes:

Séptimo ejemplo.

La Ley de Amnistía expedida por el Congreso de la Unión, publicada en el Diario Oficial de la Federación (22.I.1994) es una decisión coactiva; en efecto, en la Ley se decretó amnistía en «favor de todas las personas en contra de quienes se haya ejercitado o pudiere ejercitarse acción penal ante los tribunales del orden federal, por los delitos cometidos con motivo de los hechos de violencia, o que tengan relación con ellos, suscitados en varios municipios del Estado de Chiapas del día primero de enero de mil novecientos noventa y cuatro al día veinte del mismo mes y año, a las quince horas»; se

${ }^{7}$ Proceso No. $991 / 30$ de octubre/1995. pp. 8 y ss.

${ }^{8} \mathrm{Ni}$ Paz ni Justicia o Informe General y amplio acerca de la Guerra Civil que sufren los Choles en la Zona Norte de Chiapas, Centro de Derechos Humanos «Fray Bartolomé de Las Casas», 1996, p. 167. 
integró una Comisión que coordinaría los actos de aplicación de la Ley; los beneficios de la ley, consistentes en la extinción de las acciones y sanciones penales, se condicionaron a la entrega de rehenes y de todo tipo de armas, explosivos, instrumentos u otros objetos empleados en la realización de los delitos, en los términos que fije la Comisión, y cuando ésta declarara la cesación definitiva de los actos de hostilidad.

La ley garantiza, además, a las personas que resulten beneficiadas que «en lo futuro no podrán ser interrogadas, investigadas, citadas a comparecer, detenidas, aprehendidas, procesadas o molestadas de manera alguna por los hechos que comprende esta amnistía.»

Si la Ley de Amnistía hubiera llegado a ser eficaz el gobierno hubiera logrado sus propósitos no así el EZLN que hubiera quedado como estaba antes de la declaración de guerra; en efecto, no se hubieran satisfecho las necesidades humanas fundamentales, propósito central de las reclamaciones.

3.1.3 En el tercer tipo del modelo reactivo de poder se analiza la situación, se determinan los objetivos de A y se conocen los de B; la relación de valor entre ambos conjuntos está indeterminada al no asignárseles valor a los propósitos de $\mathrm{A}$ ni a los de $\mathrm{B}$. Se trata de un modelo paternalista en el cual A considera los propósitos de B que influyen en el resultado constituido por una decisión coactiva. siguientes:

Ejemplos que actualizan el tercer tipo del modelo reactivo de poder son los

Octavo ejemplo.

La Ley para el Diálogo, la Conciliación y la Paz Digna en Chiapas expedida por el Congreso de la Unión, publicada en el Diario Oficial de la Federación (11.III.1995); en la ley se consideran los propósitos del EZLN, al prescribir que serán objeto del acuerdo de concordia y pacificación, entre otros: I. Asegurar la paz justa, digna y duradera en el Estado de Chiapas, dentro del pleno respeto al Estado de Derecho; II. Atender las causas que originaron el conflicto y promover soluciones consensuadas a diversas demandas de carácter político, social, cultural y económico, dentro del Estado de Derecho; III. Propiciar que los integrantes del EZLN participen en el ejercicio de la política dentro de los cauces pacíficos que ofrece el Estado de Derecho, con respeto absoluto a su dignidad y garantías de ciudadanos mexicano; IV. Conciliar las demandas e intereses legítimos de los diversos sectores de la sociedad chiapaneca; V. Promover el bienestar social y el desarrollo económico sustentable en Chiapas; y VI. Proponer los lineamientos para la amnistía que, como consecuencia del proceso de diálogo y conciliación, concederá en su caso el Congreso de la Unión por los hechos relacionados con el conflicto en el Estado de Chiapas, iniciado a partir del $1^{\circ}$ de enero de 1994.» Al decidirse la suspensión de los procedimientos iniciados 
en contra de los integrantes del EZLN, como ya se explicó anteriormente y al crear la Comisión de Concordia y Pacificación (COCOPA) y la Comisión de Seguimiento y Evaluación de los Acuerdos (COSEVE) y se asume la existencia de la Comisión Nacional de Intermediación (CONAI) en tanto se establece que la COCOPA coordinará sus acciones con la instancia de mediación reconocida por los negociadores.

Se establece la obligación a los sectores públicos de apoyar el desarrollo social y económico de las comunidades indígenas y de los campesinos que lo mismo se promoverá con los sectores social y privado para fortalecer el diálogo y cooperación permanentes entre los diversos grupos de la sociedad chiapaneca; también que se fomentará la creación de fondos mixtos entre la Federación, el Estado, los municipios y el sector privado para financiar programas destinados a rescatar de la marginación a las citadas comunidades indígenas y de campesinos.

Sin embargo, la Ley es una decisión impuesta a los integrantes del EZLN, pues aun cuando responde a los objetivos del EZLN, en el artículo $1^{\circ}$ se prioriza el objetivo de buscar «la solución justa, digna y duradera al conflicto armado iniciado el $1^{\circ}$ de enero de 1994 en el Estado de Chiapas» y porque se acentúa el carácter autoritativo en la toma de decisiones al prescribir que «las disposiciones de esta Ley no impiden el ejercicio de las facultades otorgadas a las autoridades competentes y fuerzas de seguridad para que cumplan su responsabilidad de garantizar la seguridad interior y la procuración de justicia.»

Hasta aquí he tratado los modelos de poder en los cuales la autoridad toma las decisiones que son forzosas para la otra parte. Estos modelos contienen implícitamente las asunciones clásicas que han dominado el pensamiento legal: existencia de un poder con derecho a esperar obediencia, obligación moral de los otros a obedecer y legitimidad de emplear el poder y la coerción para eliminar conductas insumisas o divergentes, las cuales pueden ser suprimidas sin encontrar sus causas o sólo para resolver problemas subyacentes.

3.2 Modelos interactivos de poder. Éstos son considerados como modelos de transición entre los reactivos de poder, ya analizados, y los que resuelven problemas, que serán examinados posteriormente. Una característica de la transición es que hay negociación, aunque prevalece el mando del poder.

3.2.1 En el primer tipo del modelo interactivo de poder A y B determinan sus respectivos propósitos en una negociación directa. El valor entre los propósitos es un empate o los de $\mathrm{A}=+\mathrm{x}$ y $\operatorname{los}$ de $\mathrm{B}=-\mathrm{x}$ donde $\mathrm{x}>0.5$. En este modelo se percibe la incompatibilidad entre los propósitos de $\mathrm{A}$ y de $\mathrm{B}$, pero se reconoce que la situación está basada en el poder relativo de ambas partes y han de participar en la toma de decisión. A no puede producir el re- 
sultado y tiene que comprometerse con B a llegar a una decisión acordada (settlement agreed).

Entre los ejemplos más significativos de este modelo identifico los siguientes:

Noveno ejemplo.

El acercamiento entre el EZLN y el gobierno federal en la Catedral de San Cristóbal, del 11 de febrero al 3 de marzo de 1994. El Obispo Samuel Ruiz García desempeñó el papel de mediador; se abordaron diferentes exigencias de la Primera Declaración de la Selva Lacandona: salud, vivienda, educación, trabajo, alimentación y hubo respuestas del gobierno federal que, en principio, se consideraban satisfactorias; otras reclamaciones se calificaron de no negociables por ser de carácter nacional, así la democracia, las libertades, los procesos electorales, el reconocimiento de beligerancia al EZLN y otras se estimaron complejas como la relacionada con la autonomía de las comunidades indígenas. El Comisionado para la Paz sostuvo que los temas locales tenían salida y que «los cambios nacionales se pueden plantear por vías políticas institucionales.» El tema de la tierra fue calificado de muy complicado porque estaba en juego el equilibrio nacional. Entendía la paz como el cese de las hostilidades y solicitó al EZLN una declaración y acciones unilaterales sobre esta situación. La posición del Comisionado ubicó el problema en el Estado de Chiapas. Marcos respondió en relación con el cese unilateral: «...pensamos que la declaración debe ser bilateral. Con garantías. El cese estaría condicionado al cumplimiento de lo que se acuerde.» ...«El desarme no es al inicio, es al final del proceso de pacificación», con lo cual no estuvo de acuerdo el Comisionado.

El 23 de febrero Marcos expresó el siguiente punto de vista claro y preciso sobre el acercamiento: «...el único acuerdo a que hemos llegado es que los temas nacionales no se negocian, que la mesa de San Cristóbal está chiquita para los temas nacionales. Seguimos con el problema de la beligerancia. Ahí es el único punto en que se dice no. Todo lo demás se ha respondido. El problema es que para la decisión de unos puntos, no traemos mandato. Tenemos que regresar a consultar.» El Comisionado expresó que se esperaría el resultado de las consultas. Hubo manifestaciones de Marcos en el sentido de que lo que se llevaban era puro papel y que en los hechos, un montón de promesas; «no hay ahorita, dijo, ningún cambio de política del gobierno después el $1^{\circ}$ de enero.» Se definió por el EZLN que no había negociación sino diálogo y que de alguna manera se había logrado y que su rumbo era bueno. ${ }^{9}$

\footnotetext{
${ }^{9}$ Proceso No. 956/febrero/1995, Documento «Diálogos de San Cristóbal. Del 11 de febrero al 3 de marzo de 1994.» Cfr. Proceso No. 905/7 de marzo/1994. p. 11.
} 
Hecho determinante en la suspensión del proceso de diálogo fue el asesinato en contra de Luis Donaldo Colosio el 23 de marzo de ese año y las consecuencias económicas, sociales y políticas que produjo; entre las últimas se destacan acciones gubernamentales, de los partidos políticos y de buena parte de la ciudadanía que fijaron la atención en las campañas para Presidente de la República y en el proceso electoral del 21 de agosto del mismo año.

La dinámica del modelo conduciría a decisiones acordadas y realmente no se llegó a ninguno. El ejemplo puede actualizar este modelo o el siguiente según como se comprenda la participación del Obispo Ruiz García.

3.2.2 El otro tipo del modelo interactivo de poder presenta la intervención de una tercera parte; trae como consecuencia que los propósitos de A y B están sobre 2, pues se les asigna un valor idéntico $=(\mathrm{A}+\mathrm{B}) / 2$.

Los propósitos de ambas partes son incompatibles, pero la necesidad mutua de aceptar a la tercera parte obliga o compromete. La decisión que se obtendrá será un compromiso mediado consistente en una solución acordada (settlement agreed). La tercera persona es un mediador que busca un compromiso entre las partes o un asistente de negociación.

Actualiza el segundo tipo del modelo interactivo de poder el siguiente proceso:

Décimo ejemplo.

La negociación entre el gobierno federal y el EZLN efectuado de acuerdo con los principios de la Ley para el Diálogo, la Conciliación y la Paz Digna en Chiapas es un ejemplo del modelo interactivo de poder. En febrero de 1996 se habían logrado acuerdos en San Andrés Larráinzar entre las partes y la participación de la COCOPA y la CONAI. Los acuerdos eran válidos en virtud de las reglas de juego y estaban orientadas a lograr una reforma constitucional sobre cultura y derechos de los pueblos indios.

La COCOPA recogió los acuerdos de San Andrés Larráinzar, los redactó como una propuesta común y la presentó a ambas partes; el EZLN expresó su conformidad, pero el Dr. Zedillo Ponce de León solicitó tiempo para consultar a los constitucionalistas, estar en condiciones de expresar su voluntad y, en su caso, iniciar las acciones del proceso de reforma constitucional.

Las evaluaciones del Dr. Zedillo sobre la propuesta y sus afirmaciones públicas en diferentes foros confirman la sospecha de que aún no se comprende por el gobierno federal ni sus asesores el conflicto social hecho consciente a muchas personas a partir del $1^{\circ}$ de enero de 1994.

La respuesta del Dr. Zedillo empieza a ubicar el proceso de negociación en el modelo reactivo de poder o de control absoluto del modelo interactivo de poder, en este último al asignarle menor valor a los propósitos del EZLN. Los constitucionalistas son profesionales que dijeron al Dr. Zedillo 
lo que quería oír, opinaron al poder, desde la obediencia. Se ubicaron en las tablas viejas, diría Nietzsche. ${ }^{10}$

Parecía que los acuerdos de San Andrés Larráinzar tenían las características para ejemplificar el modelo de transición que analizamos; en efecto, hasta antes de la suspensión había conformidad de las partes negociadoras, con la participación de la COCOPA y la CONAI, sobre el contenido de las reformas constitucionales; sin embargo, todo los acuerdos se derrumbaron por decisiones unilaterales del gobierno. ${ }^{11}$

3.3 Modelo interactivo de solución de problemas. Este modelo se caracteriza porque todas las complejidades son tomadas en consideración por ambas partes.

3.3.1 En el primer tipo de modelo interactivo de solución de problemas se redefine la situación conflictiva por medio de un análisis mutuo y la participación de una tercera parte, que no es mediador ni árbitro sino facilitador, para la comprensión de la complejidad y del lenguaje utilizado por las partes, a la vez que procura acercarlas para lograr las definiciones comunes y la determinación de los propósitos también comunes. Los valores de dichos propósitos son $\mathrm{A}+\mathrm{y} \mathrm{B}+$. Se describe el modelo como el descubrimiento de propósitos comunes y la exploración de medidas acordadas para lograr su cumplimiento antes de las negociaciones; la dinámica es que se soluciona el problema bajo la intervención o el control de la tercera parte, el facilitador, mediante una resolución (resolution).

No se ha actualizado este tipo de modelo en el tratamiento del conflicto social; en efecto, no se advierte alguna situación en la cual se cumplan y, en consecuencia, no se ha intentado llegar a la solución del conflicto enfrendo las causas que lo produjeron. Además, no se advierte la presencia ni siquiera la búsqueda de un facilitador con las capacidades propias de este personaje en los conflictos sociales. En efecto, los facilitadores -que no son mediadores ni árbitros-, han de tener por función principal lograr la comprensión de las posiciones de quienes se encuentran en el conflicto y su imparcialidad no debe estar en tela de duda.

3.3.2 El segundo tipo de modelo interactivo de solución de problemas también considera todas las complejidades conocidas, A analiza la percepción de B y éste la percepción de A, actividades recíprocas que tienen como resultado la definición en común de la situación y de los propósitos. Los valores asignados a los propósitos son $\mathrm{A}+$ y $\mathrm{B}+$. Una parte importante de la

${ }^{10}$ Nietzsche, Friederich, Asi habló Zaratustra, tr. Andrés Sánchez Pascual, Alianza Editorial, S. A, España, 1981, el apartado «De las tablas viejas y nuevas», pp. 223-296.

${ }^{11}$ Para tener una idea de las diferencias entre la posición del EZLN y el gobierno federal sobre las propuestas de reforma constitucional es pertinente consultar La Jornada, del 17 de enero de 1997. 
descripción del modelo es la definición unilateral de la situación con conocimiento de la percepción opuesta, los valores prioritarios y los costos. Este modelo conduce a una solución del problema en donde el conocimiento consensual reemplaza a la tercera parte y se obtiene una resolución. analizamos.

Tampoco se ha actualizado este modelo en el tratamiento de la situación social que

\section{Valoración de las actualizaciones de los modelos}

De las ejemplificaciones anteriores derivo que los funcionarios estatales han tomado las decisiones más conservadoras para resolver el conflicto social y solamente desde la autoridad formal; la referencia de estas decisiones y actitudes ha sido el marco jurídico establecido y el de una teoría general del derecho que niega lo que podría denominarse pluralismo jurídico. ${ }^{12}$

Tal forma de actuación no atiende los reclamos relacionados con la satisfacción de necesidades humanas fundamentales de carácter material y el reconocimiento de la autonomía de los pueblos indígenas como formas de vida. Por el contrario, el gobierno federal se asume, y así actúa, en un plano de superioridad al del EZLN con referencia al poder que le otorga una legalidad conservadora y, por esa razón, se advierte la imposibilidad de que comprenda las reclamaciones hechas desde formas de vida diferentes a las que experimentan las autoridades estatales.

Además existen indicios poderosos de que las decisiones y acciones de las autoridades estatales desarrollan los modelos reactivos e interactivos de poder y que no se interesan en la solución del conflicto social. Entre algunos indicios menciono los siguientes:

a. La lista creciente de Comisionados para la Paz que, en principio, no han tenido éxito en sus gestiones; b. Las diversas sustituciones de gobernadores en el Estado de Chiapas. «En poco más de seis años ha tenido cinco gobernadores» ${ }^{13} ; \mathrm{c}$. La designación de agentes federales para que proporciones bienes y servicios, como una tarea aislada; $d$. La lucha entre gobernantes que ha producido asesinatos políticos entre los cuales se destacan los perpetrados en contra de Luis Donaldo Colosio y José Francisco Ruiz Massieu; e. La militarización, ya como guerra de baja intensidad o en las actuaciones del ENM en los primeros meses del «conflicto armado» por las cuales hubo quejas de violación de derechos humanos. $^{14}$

${ }^{12}$ Héctor Díaz Polanco, Autonomía regional. La autodeterminación de los pueblos indios, siglo veintiuno editores s.a. de c. v., México, 1991, especialmente el capítulo VI titulado «Fundamentos del sistema autonómico».

${ }^{13}$ Proceso No. 955/20/febrero/1995/p. 20.

${ }^{14}$ Consultar el trabajo terminal de Jiménez Oseguera, José Antonio y Valdivieso Martínez, Azul, titulado La Comisión Nacional de Derechos Humanos ante el conflicto en el Estado de 


\section{Un recurso válido}

Aparte de los modelos mencionados para atacar los conflictos, existen otros medios de acuerdo con John Burton y uno de ellos es la difusión de las ideas para que las conozcan las partes intervinientes y modifiquen su forma de pensar y actúen en consecuencia. Lograr este propósito sería reconocer las virtudes de una forma distinta de comprender apoyada en la fortaleza de las razones que se exponen; estas manifestaciones de ideas puede provenir de diferentes personas y grupos; así, de alguna de las partes involucradas, de otras comunidades o formas de vida en situaciones similares, de la academia, de los periodistas, de las universidades, de las organizaciones no gubernamentales, de los partidos de oposición, en fin, de los ciudadanos libres.

Ubico en este tipo de acciones las manifestaciones de protesta como la desobediencia civil y los ayunos; una que considero fue decisiva para salvar una situación dramática fue el emprendido por el Obispo Samuel Ruiz García el 19 de diciembre de $1994 . .^{15}$

La justificación para emprender acciones ciudadanas de evaluación y crítica es el principio del Estado de Derecho de los regímenes democráticos; se trata de la libre expresión de argumentos con el propósito de convencer a los oponentes de la razón que asiste a quien los expresa respecto de alguna problemática social y, en su caso, llegar a consensos racionales o a compromisos que son el resultado de la comprensión de razones.

La paz social se logrará si y sólo si existen las medidas necesarias y eficientes para disminuir, abatir o desaparecer las causas que originan el conflicto social: violación generalizada de derechos humanos y corrupción de funcionarios y ex-funcionarios públicos y existencia de grupos que disponen indebidamente de recursos humanos, materiales y financieros, así como de la estructura estatal para fines personales o de pequeños grupos relacio-

Chiapas, en el que con base en los informes de la CNDH, presentan conclusiones fundadas en relación con violaciones de derechos humanos de parte del Ejército Nacional Mexicano. Universidad Autónoma Metropolitana, Unidad Azcapotzalco, Departamento de Derecho, México, 1995.

${ }^{15}$ A principios de diciembre 1994 se tensaron las relaciones entre las partes; el Gobernador de aquella entidad federativa, Eduardo Robledo Rincón, había asumido la gubernatura el 8 de diciembre y solicitó el reforzamiento militar para «preservar la tranquilidad y las libertades constitucionales en la convivencia ciudadana» (Proceso No. 946/19 de octubre/1994, pp. 6 y ss.). La reacción a esta solicitud fue favorable; la tensión y la proximidad de una guerra o de un enfrentamiento armado se hizo inminente. Una de las reacciones a esta situación fue el ayuno en el que se comprometió el Obispo Samuel Ruiz y al que siguieron varias personas. Dicha manifestación de protesta se inició el 19 de diciembre del año en mención y lo levantó, después de quince días de sostenido, cuando consideró que había condiciones de negociación entre las partes y la amenaza de guerra se alejaba. 
nados con el poder. En consecuencia, no es por la conclusión del llamado «conflicto armado» que se acabará el conflicto social; es necesario y pertinente sanear el ambiente social con medidas integrales y no aislar los síntomas de los conflictos ni tampoco atacarlos aisladamente.

$\mathrm{Si}$ no se cumple la exigencia de sanear el ambiente será imposible tomar e instrumentar las medidas de reconocimiento de la cultura y los derechos de los pueblos indios; si las autoridades estatales tienen interés en resolver el conflicto social es necesario intervenga constructivamente atacando las causas que lo han originado y lo mantienen. 